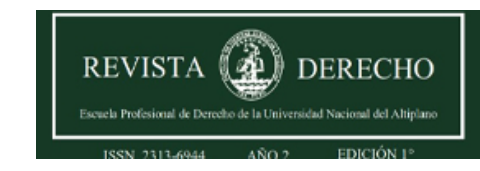

Revista de Derecho

ISSN: 2313-6944

ISSN: 2707-9651

revistaderecho@unap.edu.pe

Universidad Nacional del Altiplano

Perú

\title{
Un análisis objetivo en los últimos 5 años de la violencia doméstica en el Perú. Una Revisión Sistemática
}

Zamora Vega, Ariana Abigail; Mirabal Reynoso, Alejandro; Sosa Corrales, Carlos Rodrigo Fernando; Vargas Salas, Obed

Un análisis objetivo en los últimos 5 años de la violencia doméstica en el Perú. Una Revisión Sistemática

Revista de Derecho, vol. 6, núm. 2, 2021

Universidad Nacional del Altiplano, Perú

Disponible en: https://www.redalyc.org/articulo.oa?id=671870938007

DOI: https://doi.org/10.47712/rd.2021.v6i2.141

\section{(c) (1)}

Esta obra está bajo una Licencia Creative Commons Atribución 4.0 Internacional. 
Artículos de naturaleza variada

\title{
Un análisis objetivo en los últimos 5 años de la violencia doméstica en el Perú. Una Revisión Sistemática
}

\author{
An objective analysis in the last 5 years of domestic violence in Peru. A Systematic Review \\ Ariana Abigail Zamora Vega \\ Universidad Católica de Santa María, Perú \\ ariana.zamora@ucsm.edu.pe \\ DOI: https://doi.org/10.47712/rd.2021.v6i2.141 \\ Redalyc: https://www.redalyc.org/articulo.oa? \\ id $=671870938007$
}

iD https://orcid.org/0000-0002-8832-7213

Alejandro Mirabal Reynoso

Universidad Católica de Santa María, Perú

alejandro.mirabal@ucsm.edu.pe

iD https://orcid.org/0000-0001-9056-0893

Carlos Rodrigo Fernando Sosa Corrales

Universidad Católica de Santa María, Perú

carlos.sosa@ucsm.edu.pe

iD https://orcid.org/0000-0002-5526-608X

Obed Vargas Salas

Universidad Católica de Santa María, Perú

ovargas@ucsm.edu.pe

iD https://orcid.org/0000-0003-1084-3477

Recepción: 17 Julio 2021

Aprobación: 26 Agosto 2021

Publicación: 14 Septiembre 2021

\section{RESUMEN:}

Objetivo: Identificar sistemáticamente la evidencia científica publicada en revistas indexadas en los últimos años sobre la violencia doméstica y analizar aspectos relevantes para contrastar con la legislación peruana. Método: Se realizó la búsqueda y revisión manual de publicación científica en 7 bases de datos. El número total de artículos obtenidos a partir de las búsquedas ha sido de seiscientos veinte mil seiscientos veintiocho $(620$ 628) de los cuales, después de aplicar los criterios de inclusión y exclusión se seleccionaron dieciocho (18). Resultados: Del análisis y discusión de cada uno de los artículos se evidenció la relevancia actual que comprende la estadística de la violencia doméstica, resultando en afectar emocionalmente para el desarrollo de la integridad de la víctima; las repercusiones posteriores demuestran una necesidad de enfrentar la problemática desde distintos ámbitos, aparte del legal, que si bien es demostrada su ineficacia para combatir el ya mencionado problema en la jurisdicción peruana, se pueden evaluar distintas otras variables que resultan en una acepción más amplia del tema, que, objetivamente proveerá de mejores soluciones.

Palabras clave: Violencia, Familia, Violencia doméstica.

\section{Abstract:}

Objective: Identify systematically, the scientific evidence published in recent years indexed journals about domestic violence and analyze relevant aspects to contrast with Peruvian legislation. Method: The search and scientific publishing manual revision was made in 7 different reference databases: SCOPUS, WEB OF SCIENCE, PUBMED, VILEX, DIALNET, SCIENCEDIRECT y GOOGLE SCHOLAR. The total number of obtained articles from the searches, has been of six hundred twenty thousand six hundred and twenty-eight (620 628) of which, after applying our inclusion and exclusion criteria, were selected eighteen (18). Results: From the analysis and discussion of every article, it was evidenced the actual relevance that comprehends the domestic violence statistics, resulting to affect emotionally the victim's integrity; the subsequent repercussions demonstrate a necessity to front face the problematic from different scopes, besides legal aspects; that although its inefficacy to face this problem in the 
Peruvian jurisdiction has been proved, it can be evaluated other variables that could result in a wide better formulated meaning and understanding, that, actually will provide better solutions.

KEYwORDS: Violence, Family, Domestic Violence.

\section{INTRODUCCIÓN}

En esta abstracción encontramos definiciones más específicas que concretan un entendimiento mejor del tema a investigar y aportan de igual manera una referencia bibliográfica más amplia que sustente diversas premisas establecidas por la teoría.

En objeto, la violencia puede ser física, tanto como psicológica y a su vez tanto como arma de clase (según Marx), tanto como represiva legal (según W. Benjamin), tanto como sistemática (según Zizek) o inherente al lenguaje (según Chomsky), al ser un fenómeno social de causas primitivas parte de la naturaleza del ser humano; suele ser un recurso para explicar diversos factores conductuales a los cuales la racionalidad no puede apelar, la violencia en sí, es un impulso primitivo para reprimir una actitud contraria; esta definición puede ser complementada por estudios que la complejizan a través de investigaciones sociológicas/psicológicas. No obstante, la trascendencia de la violencia en diferentes conductas humanas más civilizadas, ha sido internalizada y absorbida por la propia sociedad, provocando la normalización de conductas violentas dentro de, contextualizando, la familia en el ámbito doméstico. Ciertas de estas acepciones serán revisadas dentro de este mismo marco teórico complementado por el elemento jurídico completando así una causalidad del estudio del tema dentro de la legislación peruana.

En esencia, la revisión pretende analizar fuentes de la legislación peruana que son activos directos en la regulación y lucha contra la violencia doméstica; en la vida republicana del país, se han arraigado, incluso a la Constitución, diversas irregularidades propias de la sociedad patriarcal, avalando muchas de las conductas machistas más normalizadas incluso dentro del ámbito jurídico, revisamos fuentes actuales que proporcionan la información necesaria para evaluar el impacto de las mismas en la problemática y la trascendencia a futuro que estas puedan tener.

\section{Definición de Violencia}

La violencia, en su concepción más básica puede entenderse como (citando a la ONU) "El uso intencional de la fuerza o el poder físico, de hecho o como amenaza, contra uno mismo, otra persona o un grupo o comunidad, que cause o tenga muchas probabilidades de causar lesiones, muerte, daños psicológicos, trastornos del desarrollo o privaciones", es una racionalización concreta que engloba de manera general los aspectos que envuelven a la violencia como tal, describiendo la causalidad de la misma (causas - efectos). Pero trasciende a esto una visión más específica que mediante abstraerse en un análisis filosófico de la materia podemos encontrar definiciones que yacen diversos tipos que involucran tipos de violencia más abstracta referidos al sistema, como en el ensayo "Para Una Crítica a la Violencia" de Walter Benjamin; donde se es referido un tipo de violencia tácito inherente al funcionamiento del sistema legal o en palabras del filósofo esloveno Slavoj Zizek, algo arraigado a una conducta normalizada por la sociedad.

\section{DEFINICIÓN DE VIOLENCIA INTRAFAMILIAR/DOMÉSTICA}

Según (Nizama Valladolid, 2019). Cuando hablamos del término intrafamiliar, podemos abarcar una amplia gama de violencia que puede ocurrir entre los participantes de la familia, como entre padres e hijos, entre cónyuges, etc. En resumen, mientras haya dos o más miembros en la familia, puede haber violencia entre ellos. Cuando ocurre un incidente de violencia intrafamiliar, este restringe y reprime el comportamiento de las 
personas afectadas y su crecimiento como seres humanos, ignora su dignidad, destruye su voluntad e interfiere con su acceso al conocimiento. En situaciones de violencia doméstica, muchos expertos señalan que tienen un carácter cultural, antropológico o social. Sin embargo, en estos casos, los motivos que suelen ser más fáciles de detectar o enfatizar son el síndrome de Othello (comportamiento excesivo de fibra de vidrio), alcoholismo, TDI (trastorno de identidad), drogadicción, pobreza, etc. Para tratar estos casos de violencia doméstica en general, las personas deben ser educadas desde dentro de la familia hasta que puedan adaptarse nuevamente a una vida social saludable.

Varios estudios de la Organización Mundial de la Salud han demostrado que la violencia doméstica está relacionada con varios problemas graves y riesgos para el bienestar de las víctimas. A nivel psicológico, esto puede conducir a un desequilibrio psicológico en la víctima y las personas que conviven con ella (generalmente niños).

UNICEF también afirmó que no tiene que estar en un lugar específico llamado "hogar" para ser llamado violencia intrafamiliar, sino solo por otro miembro de la familia contra uno de los miembros de la familia.

Así como hemos visto claramente que las condiciones para que ocurra la violencia doméstica son físicas y psicológicas, a continuación, aprenderemos más y cómo se manifiestan.

\section{Tipos DE VIOLENCIA}

\section{Violencia Física}

Según (CONGRESO DE LA REPÚBLICA, 2015a, p. artículo 8 Ley 30364, inciso a). La violencia física es la acción o conducta, que causa daño a la integridad corporal o a la salud. Se incluye el maltrato por negligencia, descuido o por privación de las necesidades básicas, que hayan ocasionado daño físico o que puedan llegar a ocasionar, sin importar el tiempo que se requiera para su recuperación.

\section{Violencia psicológica}

Según (CONGRESO DE LA REPÚBLICA, 2015a, p. artículo 8 Ley 30364, inciso b).La violencia psicológica, que por lo general acompaña a las otras formas de violencia y que también tiene como base la desigualdad de género, es definida en la Ley $\mathrm{N}^{\circ} 30364$ como "la acción o conducta tendiente a controlar o aislar a la persona contra su voluntad, a humillarla o avergonzarla y que puede ocasionar daños psíquicos. Daño psíquico es la afectación o alteración de algunas de las funciones mentales o capacidades de la persona, producida por un hecho o un conjunto de situaciones de violencia, que determina un menoscabo temporal o permanente, reversible o irreversible del funcionamiento integral previo"

\section{Metodología}

Diseño

El cubrir los objetivos descritos nos ha llevado a realizar una búsqueda manual en 7 bases de datos de referencia: SCOPUS, WEB OF SCIENCE, PUBMED, VILEX, DIALNET, SCIENCEDIRECT y GOOGLE SCHOLAR. La búsqueda se realizó desde marzo de 2021 hasta junio de 2021. Se revisaron el título, el resumen y el texto completo para su inclusión. Los autores utilizaron la estrategia PICO para describir todos los componentes relacionados a nuestra investigación. Ver anexo 1. 
Anexo 1

\begin{tabular}{|l|l|}
\hline $\begin{array}{l}\text { PACIENTE CON } \\
\text { PROBLEMA DE } \\
\text { INTERÉS }\end{array}$ & $\begin{array}{l}\text { ¿El artículo evalúa a las víctimas de violencia } \\
\text { intrafamiliar /doméstica en Latinoamérica? ¿El artículo evalúa a } \\
\text { perpetradores de violencia intrafamiliar /doméstica? }\end{array}$ \\
\hline INTERVENCIÓN & $\begin{array}{l}\text { ¿Hace alusión a las intervenciones que se dan frente a un caso de } \\
\text { violencia intrafamiliar /doméstica? ¿Hace alusión a alguna política } \\
\text { o medida frente a la violencia intrafamiliar /doméstica en el } \\
\text { contexto del covid19? }\end{array}$ \\
\hline COMPARACIÓN & $\begin{array}{l}\text { ¿Compara cifras de denuncias en los diferentes países de } \\
\text { Latinoamérica? ¿Compara la efectividad de las medidas tomadas } \\
\text { para el control o disminución de cifras? }\end{array}$ \\
\hline $\begin{array}{l}\text { OUTCOME } \\
\text { Variable que mide } \\
\text { el resultado de } \\
\text { interés) }\end{array}$ & $\begin{array}{l}\text { ¿Aporta información estadística actualizada de violencia durante } \\
\text { la cuarentena? ¿Brinda información sobre aspectos relevantes en } \\
\text { la violencia intrafamiliar idoméstica? }\end{array}$ \\
\hline
\end{tabular}

La calidad metodológica se evaluó mediante la lista de verificación de revisión sistemática PRISMA.

La estrategia de búsqueda utilizada en el presente trabajo se ha estructurado en búsquedas independientes a partir de la combinación de diferentes términos de búsqueda. Esta fórmula ha sido la escogida debido a que en primera instancia y después de elaborar diferentes formatos de búsqueda más completos y utilizando operadores como el OR o el AND o ambos juntos, se identificó una pérdida de resultados que consideraba importante. Esto lo atribuyó al funcionamiento de algunas de las bases de datos que mostraban resultados muy dispares dependiendo del operador de búsqueda o fórmula que utilizase.

Términos de búsqueda en castellano.

$((((((((($ familia) $\mathrm{O}$ (parentesco)) O (cónyuge) $) \mathrm{O}$ (casado)) OR (hijo)) AND (maltrato $))$ OR (violencia)) OR (abuso)) OR (agravio))

Términos de búsqueda en inglés.

$((((((((($ family) OR (kinship)) OR (spouse)) OR (married)) OR (son)) AND (mistreatment $)))$ OR (violence)) OR (abuse)) OR (grievance))

Se excluyeron de documentos duplicados, documentos que superen el límite de años desde su publicación, documentos que tengan un enfoque de género, artículos en que no contengan un enfoque global o de Perú, artículos que no cuenten con open Access, artículos con temática "Clínica" o "Médica". Se incluyeron artículos que estudien a las víctimas por violencia doméstica / intrafamiliar, artículos que estudien a perpetradores de violencia doméstica / intrafamiliar, artículos que contengan un enfoque global, artículos que contengan un enfoque en Perú, artículos con open Access, artículos publicados entre 2016 - 2021, artículos de contenidos en temática de "ciencias sociales" o "ciencias jurídicas", artículos de investigación, artículos de revisión, en idioma: Inglés - Español, adicionalmente fragmentos de libros y leyes, decretos dentro de la normativa Peruana.

El número total de artículos obtenidos a partir de las búsquedas ha sido de seiscientos veinte mil seiscientos veintiocho (620 628) de los cuales, después de aplicar los criterios de inclusión y exclusión se han seleccionado dos mil seiscientos veintinueve (2629). En la primera búsqueda, en la estrategia de búsqueda se elimina duplicados.

Se obtuvo un total de siete artículos (7), de los cuales, fueron seleccionados un total de dos mil seiscientos veintidós (2622) artículos. En la segunda búsqueda, la estrategia se centró en filtrar los títulos más relevantes, desechando los no necesarios. En este caso, el número de artículos obtenidos fue de dos mil quinientos setenta y cinco (2575) y los seleccionados cincuenta

Anexo 2. y cuatro (54). En la tercera búsqueda, 
la estrategia se centró en la revisión de los artículos a profundidad para verificar que su contenido colabore con la realización de nuestro objetivo. Se obtuvieron treinta y seis (36) artículos de los que se seleccionaron dieciocho (18). Ver Anexo 2.

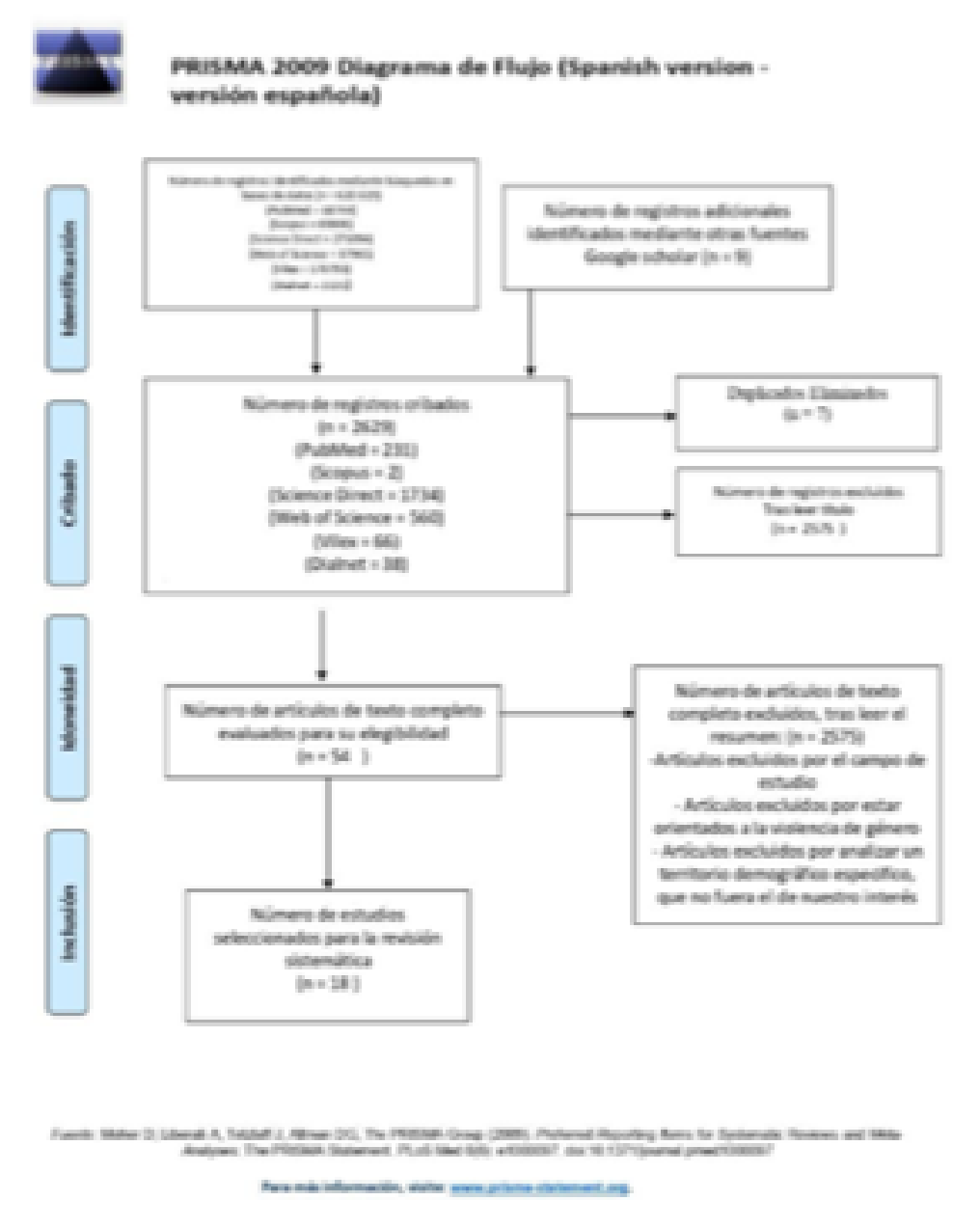

Anexo 2.

\section{RESULTADO}

Se evaluaron nueve artículos científicos que cumplieron rigurosamente los criterios de inclusión. Adicionalmente se identificaron dentro de la normativa peruana, una ley y dos decretos.

Del análisis se evidenció la preocupación en relación con el tema propuesto. A continuación de manera descriptiva se presenta en la tabla los análisis. 


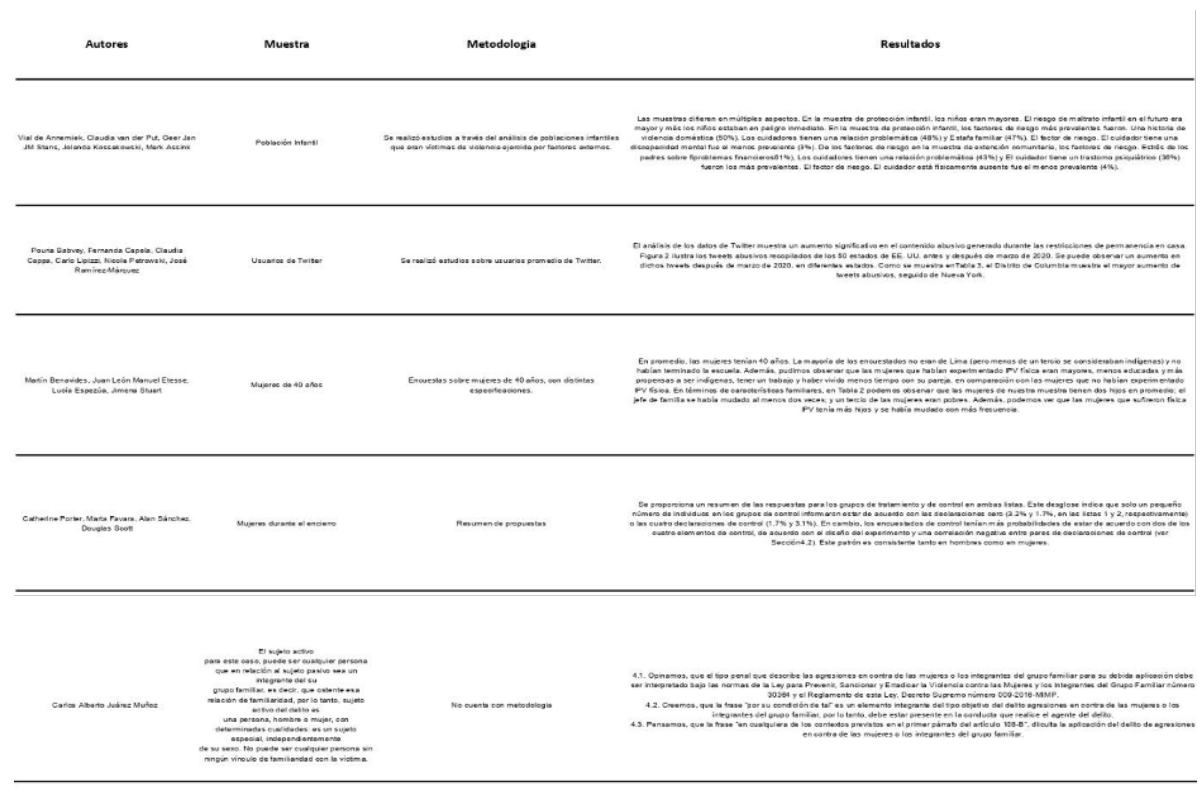

El artículo de Vial de Annemiek, Claudia van der Put, Geer Jan JM Stans, Jolanda Kossakowski y Mark Assink, investigaron los factores de riesgo del maltrato en niños y cómo se interrelacionan, tomando como muestra familias de alto riesgo y de riesgo medio en EE UU. Concluyendo en los resultados demostrando el impacto psicológico que experimenta el niño en casos de abuso infantil.

En el artículo Pouria Babvey, Fernanda Capela, Claudia Cappa, Carlo Lipizzi, Nicole Petrowski, José Ramírez-Márquez, mediante la elaboración de una investigación sistemática lograron detallar cómo es que las redes sociales afectan la exposición a la pandemia. Y se concluye la disociación de la persona en los medios modernos que favorece en la incidencia de casos de violencia sistemática.

El artículo de Martín Benavides, Juan León Manuel Etesse, Lucía Espezúa, Jimena Stuart, quienes mediante una encuesta realizaron una investigación sistemática acerca de la violencia intrafamiliar y recogió además la demografía del barrio donde vivían. Sustentando en las conclusiones, en la premisa de la idea del estatus como individuo de la mujer, que afecta directamente a lo propensa que está la misma al abuso.

En artículo de Catherine Porter, Marta Favara, Alan Sánchez, Douglas Scott, se concentran en realizar un análisis cuantitativo de la violencia física familiar durante la pandemia del covid, usando de muestra a las familias peruanas que sufrieron violencia doméstica entre los 26 meses de encierro por COVID. La evidencia se encuentra en la normalización sistemática de la violencia contra la mujer, donde incluso ambos géneros son propensos a aceptar premisas que involucran aceptar conductas machistas.

En el artículo de Barrios Montalvo, Patricia Elena realiza un análisis cualitativo acerca de cómo aumenta la incidencia de los casos y como de la violencia familiar y las repercusiones que causa. Usando de muestra los casos ocurridos en la provincia de Acobamba, basa los estudios en la pasión y el desenfreno que llevan no tener un alma libre, y este se puede ver como el punto de inicio de los problemas dentro de la familia, esto suele ser muy pocas veces visto.

En el artículo de Céspedes Cajo, Cynthya Fiorella realiza un análisis acerca de la institución de la familia, usando como muestra a la familia del Perú y la legislación que se encuentra acerca de la misma. Concluye que dentro de eso los aspectos que posee esta importante institución que continuamente evoluciona y existe jurisprudencia acerca de varias notas sobre esta temática.

En el artículo de Barragán García, José Gabriel corresponde a un artículo de investigación en el que evalúan el impacto de la emergencia sanitaria en razón del derecho de la familia, usando como muestra a familias de América Latina. Además, concluyen que deben reconocer que durante esta crisis sanitaria los más afectados 
vienen siendo los menores de edad por lo que se les debe dar un mejor cuidado, viendo como los procesos que se debían dar con los padres separados afectan a los menores, así como las discusiones interfamiliares.

En el artículo Bermúdez Tapia, Manuel corresponde a una investigación acerca de la responsabilidad judicial dentro de los procesos judiciales que incluyen violencia familiar en el Perú, tuvo como muestra la jurisprudencia hallada de varios procesos judiciales. Se concluyó que el factor judicial afecta demasiado al momento de la evaluación de casos de violencia intrafamiliar, debido a que si se juzga de manera errónea por negligencia esto afecta aumentando la incidencia y la manera en la que se presentan los hechos.

En el artículo de Juárez Muñoz, Carlos Alberto corresponde a una investigación detallada acerca de la legislación del Perú; tuvo como muestra la normativa nacional vigente. Se concluyó que se debe seguir lineamientos los cuales ayuden a erradicar la violencia doméstica, teniendo en cuenta que durante los procesos se encontrarán muchas trabas dentro de las mismas leyes, por lo que se debe tomar en cuenta la conducta que tuvo el agresor.

\section{LEY Na 30364}

LEY PARA PREVENIR, SANCIONAR Y ERRADICAR LA VIOLENCIA CONTRA LAS MUJERES Y LOS INTEGRANTES DEL GRUPO FAMILIAR

Artículo 1 . Objeto de la Ley La presente Ley tiene por objeto prevenir, erradicar y sancionar toda forma de violencia producida en el ámbito público o privado contra las mujeres por su condición de tales, y contra los integrantes del grupo familiar; en especial, cuando se encuentran en situación de vulnerabilidad, por la edad o situación física como las niñas, niños, adolescentes, personas adultas mayores y personas con discapacidad. Para tal efecto, establece mecanismos, medidas y políticas integrales de prevención, atención y protección de las víctimas, así como reparación del daño causado; y dispone la persecución, sanción y reeducación de los agresores sentenciados con el fin de garantizar a las mujeres y al grupo familiar una vida libre de violencia asegurando el ejercicio pleno de sus derechos.

\section{DECRETO LEGISLATIVO № 1408}

Artículo 1.- Objeto

El presente Decreto Legislativo tiene por objeto desarrollar el marco normativo para el diseño, implementación, seguimiento, evaluación e institucionalización de servicios especializados, en articulación con los tres niveles de gobierno, para el fortalecimiento y la prevención de la violencia en las familias, mediante acciones y medidas dirigidas a identificar factores de riesgo, gestionar los conflictos, erradicar la discriminación y la violencia entre sus integrantes, que afectan su convivencia pacífica, democrática y respetuosa.

DECRETO LEGISLATIVO N. ${ }^{\circ} 1386$

\section{Artículo 1.- Objeto}

El presente Decreto Legislativo tiene por objeto fortalecer la prevención, erradicación y sanción de toda forma de violencia contra las mujeres y contra los integrantes del grupo familiar, mejorando los mecanismos de atención y protección de las víctimas, especialmente el marco que regula las medidas de protección.

\section{DISCUSIÓN}

En el análisis de artículos, se encuentra que el tema central provee distintos enfoques sobre la causa de la violencia y conflictúan en las posibles soluciones al mismo, se entiende la naturaleza violenta del hombre y la raíz sistemática del problema, más posteriores artículos sustentan la viabilidad de los mecanismos estatales para garantizar la correcta forma de afrontar la problemática.

En análisis particular argumentamos la necesidad de reconocer la incapacidad de estos mismos mecanismos para reducir las alarmantes estadísticas en torno al tema. Como puede ser visto a lo largo de la investigación, distintas causas que evocan en el resultado estadístico de las alarmantes cifras de violencia doméstica, yacen arraigadas a problemas que no solo atraviesan por la administración estatal, resultando una problemática mucho más compleja.

Partiendo desde otra perspectiva, abordar la violencia desde un ámbito legal, como fue antes mencionado, resulta insulso en un entendimiento real, puesto que dicho ámbito está sujeto de igual manera a cambios sociales que avalen el procedimiento jurídico; la sociedad ajusta una moral común bajo la cual se normalizan 
actitudes, en este contexto, el cambio generacional es vital para establecer un impacto en la idiosincrasia general, por lo que concluimos la necesidad de un plan integral de lucha contra la violencia.

\section{CONFLICTO DE INTERESES}

Los autores no reportan conflicto de intereses. Los autores son los únicos responsables del contenido y la redacción del artículo.

\section{CONCLUSIÓN}

Después de haber evaluado las distintas fuentes presentadas, se puede concluir que:

Que la responsabilidad judicial en un caso de violencia familiar es muy alta, pues si existiera un caso de negligencia provocaría consecuencias peores que por las que inicialmente se inició el juicio. Por lo que aumentaría el punto de aparición dentro del caudal de las pasiones desborda al orden de la razón, debemos evadir aislar las problemáticas, para poder plantear una solución que abarque tanto lo social y conductual como lo jurídico y legal.

Que las leyes deben ser ejercidas con el fin de erradicar, disminuir y sancionar a las personas teniendo en cuenta el objetivo que se tuvo al realizar el acto que atente contra la célula básica del estado, que viene siendo la familia, puesto que, dentro de la legislación peruana para discernir esta problemática, resulta ineficiente la administración de esta y trae consigo efectos colaterales en la sociedad que padece demostrado en los altos índices de violencia doméstica.Por lo que debemos entender la problemática como ciertas acepciones aisladas es un sesgo interpretativo y concluye un conflicto de razonamiento y debido a la necesidad de este mismo razonamiento complejo e integral.

\section{BIBLIOGRAFÍA}

Zizek, S. (2007). Sobre la Violencia. PAIDÓS Esenciales. Obtenido de https://www.planetadelibros.com/libros_con tenido_extra/37/36667_SOBRE_LA_VIOLENCIA.pdf

Benjamin, W. (1921). Para una Crítica de la Violencia. UNAM. Obtenido de https://versionojs.xoc.uam.mx/index. $\mathrm{php} / \mathrm{version} /$ article/download/497/495\#page $=10$.

Alan Sánchez, C. P. (s.f.). The impact of COVID-19 lockdowns on physical domestic violence: Evidence from a list randomization experiment. GRADE. Obtenido de https://www.grade.org.pe/publicaciones/the-impact-of-co vid-19-lockdowns-on-physical-domestic-violence-evidence-from-a-list-randomization-experiment/.

BARRIOS MONTALVO, P. E. (2019). FACTORES BIOLÓGICOS, PSICOLÓGICOS Y SOCIALES QUE INCIDEN EN EL INCREMENTO DE LA VIOLENCIA FAMILIAR Y SU REPERCUSIÓN EN LOS DERECHOS HUMANOS. REPOSITORIO DE LA UNIVERSIDAD NACIONAL FEDERICO VILLAREAL, 202. Obtenido de http://repositorio.unfv.edu.pe/bitstream/handle/UNFV/3627/BARRIOS\% 20MONTALVO\%20PATRICIA\%20ELENA\%20-\%20DOCTORADO.pdf?sequence=1\&isAllowed=y

Narváez, M. L. (2017). LA TUTELA DE PREVENCIÓN EN LOS PROCESOS POR VIOLENCIA FAMILIAR. IUS ET VERITAS. doi:https://doi.org/10.18800/iusetveritas.201702.008

Juárez Muñoz, C. (2020). El delito de agresiones en contra de las mujeres o integrantes del grupo familiar. LEX REVISTA DE LA FACULTAD DE DERECHO Y CIENCIAS POLÍTICAS, 18(26), 321-346. doi:http:// dx.doi.org/10.21503/lex.v18i26.2182

Bermúdez Tapia, M. (2020, julio). Revista Exégesis - Books and Journals. vLex. https://vlex.com.pe/source/revista-e xegesis-30919 
Barragán García, J. G. (2020, julio). El impacto de la emergencia sanitaria en el derecho de familia: la afectación de las relaciones parentales indispensables. vLex. https://vlex.com.pe/vid/impacto-emergencia-sanitaria-derecho850692474

Babvey, P., Capela, F., Cappa, C., Lipizzi, C., Petrowski, N., \& Ramirez-Marquez,J. (2021). Using social media data for assessing children's exposure to violence during the COVID-19 pandemic. Child Abuse \& Neglect, 116, 104747. https://doi.org/10.1016/j.chiabu.2020.104747

Benavides, M., León, J., Etesse, M., Espezúa, L., \& Stuart, J. (2019). Exploring the association between segregation and physical intimate partner violence in Lima, Peru: The mediating role of gender norms and social capital. SSM Population Health, 7, 100338. https://doi.org/10.1016/j.ssmph.2018.100338

CONGRESO DE LA REPÚBLICA. (2015a). Ley 30364. Diario El Peruano. https://busquedas.elperuano.pe/norm aslegales/ley-para-prevenir-sancionar-y-erradicar-la-violencia-contra-ley-n-30364-1314999-1/

CONGRESO DE LA REPÚBLICA. (2015b). LEY PARA PREVENIR, SANCIONAR Y ERRADICAR LA VIOLENCIA CONTRA LAS MUJERES Y LOS INTEGRANTES DEL GRUPO FAMILIAR. Diario El Peruano. https://busquedas.elperuano.pe/normaslegales/ley-para-prevenir-sancionar-y-erradicar-la-violencia-c ontra-ley-n-30364-1314999-1/

Congreso de la República. (2018). DECRETO LEGISLATIVO QUE MODIFICA LA LEY N. 30364, LEY PARA PREVENIR, SANCIONAR Y ERRADICAR LA VIOLENCIA CONTRA LAS MUJERES Y LOS INTEGRANTES DEL GRUPO FAMILIAR. Diario El Peruano. https://busquedas.elperuano.pe/normaslega les/decreto-legislativo-que-modifica-la-ley-n-30364-ley-para-p-decreto-legislativo-n-1386-1687393-4/

Vial, A., van der Put, C., Stams, G. J. J., Kossakowski, J., \& Assink, M. (2020). Exploring the interrelatedness of risk factors for child maltreatment: A network approach. Child Abuse \& Neglect, 107, 104622. https://doi.org/10 $.1016 /$ j.chiabu.2020.104622

Nizama Valladolid, M. (2019). Enfoque criminológico de la violencia de género e intrafamiliar èes eficaz la respuesta penal? Vox juris, 37(1), 67-78. https://doi.org/10.24265/voxjuris.2019.v37n1.06

Walton, S. M., \& Pérez, C. A. S. (2019). Intrafamily violence. A current health problem. Gaceta Médica Espirituana, 21(1), 96-105

Pérez Pita, D. C., Vassallo Cruz, K. L., \& Burga Coronel, A. M. (2014). Escasa efectividad del marco legal sobre violencia familiar en la realidad peruana:" mucho ruido y pocas nueces".

Urrútia, G., \& Bonfill, X. (2010). Declaración PRISMA: una propuesta para mejorar la publicación de revisiones sistemáticas y metaanálisis. Medicina clinica, 135(11), 507-511. 